\title{
STUDIES IN THE DYNAMICS OF DISINFECTION
}

\section{THE REACTION BETWEEN PHENOL AND BACT. COLI: THE EFFECT OF TEMPERATURE ON THE USUALLY ACCEPTED CONCENTRATION EXPONENT AND THE CALCULATION OF A MORE SATISFACTORY EXPONENT BASED ON THEORETICAL CONSIDERATIONS}

\author{
By R. C. JORDAN, Ph.D. and S. E. JACOBS, Ph.D. \\ From the Physiology Department, University College of South Wales and Monmouthshire, \\ Cardiff, and the Bacteriological Laboratory, Imperial College of Science and Technology, London
}

(With 4 Figures in the Text)

The relationship between the concentration of a germicide and the time required to reach a given degree of mortality amongst the cells of a population of bacteria exposed to its action is one of profound importance. In an earlier paper of this series (Jordan \& Jacobs, 1944) it was shown that at $35^{\circ} \mathrm{C}$. the virtual sterilization time (v.s.t.) for standardized cultures of Bact. coli exposed to phenol under rigidly controlled conditions was determined by the concentration $(C)$ in accordance with the formula $C^{n} \times t=K$, where $t$ is the v.s.t. and $n$ and $K$ are constants. This was in agreement with the findings of other workers. Subsequently further experiments with phenol and Bact. coli were performed at temperatures other than $35^{\circ} \mathrm{C}$. (Jordan \& Jacobs, 1945), and it therefore became possible to examine the effect of temperature on the relation between $v . s . t$. and concentration, an aspect of disinfection which hitherto has received little attention.

\section{RESULTS}

The further experiments referred to above were originally designed to elucidate the effect of temperature on the v.s.t. at constant phenol concentration, and no attempt was made to employ identical temperatures at each of the concentrations tested. Consequently, the data obtained could only be used in a study of the effect of varying the concentration at constant temperature after the mathematical relationship between $v$.s.t. and temperature at constant concentration had been worked out. It appeared (Jordan \& Jacobs, 1946a) that this latter relationship was complex and not adequately expressed by any of the formulae usually employed in the calculation of temperature coefficients. However, consideration of the type of relationship theoretically to be expected between $v . s . t$. and temperature led to the expectation that the formula of the Pearl-Verhulst logistic curve
(Pearl, 1930) would fit the data more closely. In fact this proved to be the case, a very satisfactory fit being obtained with this one type of formula at all phenol concentrations (Jordan \& Jacobs, 1946b). From the several equations expressing the relationship between v.s.t. and temperature at different phenol concentrations, values for the $v$.s.t. at chosen temperatures could readily be calculated. The data thus obtained are presented in Table 1, but a number of these values (those which are indicated by asterisks) fall outside the range of temperature and concentration covered experimentally. Fig. 1 shows the graphs of v.s.t. plotted against concentration for all the temperatures chosen except for parts of the curves at $15^{\circ} \mathrm{C}$. and $20^{\circ} \mathrm{C}$, , the remaining data for which would not fit conveniently into the same figure with the rest.

When the calculated values of the v.s.t. which fall within or only slightly outside the range covered experimentally are tested for their agreement with the formula $C^{n} \times t=K$ they are found to fit very well, since the graphs of $\log (v$.s.t. $)$ plotted against $\log C$ are linear (Fig. 2). Table 2 gives the slopes of the calculated best fitting straight lines with their standard errors and it is clear that the latter are all satisfactorily small. The graphs in Fig. 2 correspond to the calculated regression lines and the magnitudes of their slopes are the values of the concentration exponent $n$ in the above formula. The value for $n$ at $35^{\circ} \mathrm{C}$. of 5.9296 \pm 0.4488 is very close to that of $5 \cdot 5877 \pm 0 \cdot 1599$ obtained directly from the experimental data for $35^{\circ} \mathrm{C}$. over a wider range of concentration (Jordan \& Jacobs, 1945). Evidently from $20^{\circ}$ to $35^{\circ} \mathrm{C} . n$ can be regarded as constant. This is in accordance with the conclusion reached by Tilley (1939) who, working with phenols and alcohols, found that the value of $n$ is characteristic of an organism and a germicide and is unaffected by temperature within wide limits. However, the present data show that at $39^{\circ} \mathrm{C}$. the value of $n$ is higher than 
at $35^{\circ} \mathrm{C}$, the difference being significant, although only just so $(P=0 \cdot 02-0 \cdot 05)$.

\section{DISCUSSION}

The values of the concentration exponents derived from the formula $C^{n} \times t=K$ are very satisfactorily constant over a wide range of temperature and for many purposes this method of treating disinfection data can be considered adequate and extremely useful. But it is also true that this fomula can at best be only an approximation, since it is well known that there is concentration range of a germicide, account must be taken of these threshold considerations. Reichel (1909) realized this and employed the formula

$$
\left(C-C_{0}\right)^{n} \times t=K,
$$

where $C_{0}$ represents the threshold concentration. However, in the present work it is clear that since the formula $C^{n} \times t=K$ fits the data so successfully over the experimental range, the modified formula of Reichel cannot also apply unless $C_{0}$ is very small. But the threshold concentration is evidently not small, at any rate at low temperatures (see Fig. 1), and it seems

Table 1. Calculated values of the v.s.t. derived from formulae relating v.s.t. to temperature at constant phenol concentration, together with their logarithms and $\log _{10}($ v.s.t. -10$)$

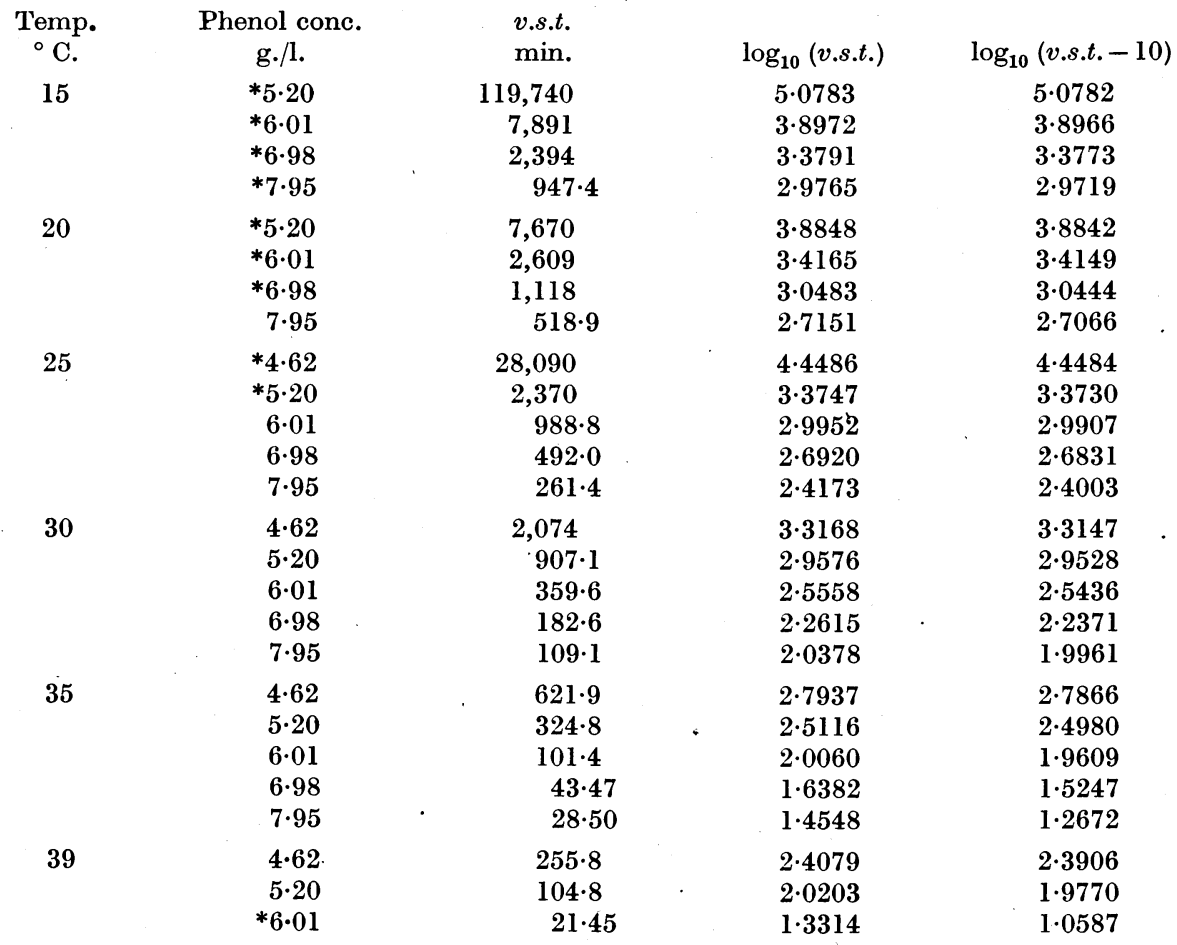

* These values fall outside the experimental range of temperature and concentration.

a threshold value for the concentration of a drug below which no effect is produced. If this is also true of bactericidal action, then, as Rahn (1932) has stressed, $t$ must become infinitely large while $C$ is still measurable. Accordingly, $n$ must increase as $C$ approaches the threshold value and some evidence that this does occur was secured from experiments on the disinfection of Bact. coli cultures with phenol at $35^{\circ} \mathrm{C}$. (Jordan \& Jacobs, 1944, 1945). From the shapes of the graphs in Fig. 1 it is clear that these minimum or threshold concentrations do exist and that they vary with the temperature. In any attempt to find an expression which will be valid over the complete
Table 2. Showing the relationship between temperature and concentration exponent

Concentration ex-

ponent $(n)$, i.e. the Ratio of slope of regression of Concentration $n$ to its

Temp. $\log _{10}$ (v.s.t.) on range standard

${ }^{\circ}$ C. $\log _{10}$ (concentration) g./l. error

$20 \quad 5 \cdot 7701 \pm 0 \cdot 0669 \quad 6 \cdot 01-7 \cdot 95 \quad 86 \cdot 2$

$25 \quad 5 \cdot 1397 \pm 0 \cdot 2179 \quad 5 \cdot 20-7 \cdot 95 \quad 23 \cdot 6$

$30 \quad 5 \cdot 4173+0 \cdot 3689 \quad 4 \cdot 62-7 \cdot 95 \quad 14 \cdot 7$

$35 \quad 5 \cdot 9296 \pm 0 \cdot 4488 \quad 4 \cdot 62-7 \cdot 95 \quad 13 \cdot 2$

$39 \quad 9 \cdot 4746 \pm 0 \cdot 9716 \quad 4 \cdot 62-6 \cdot 01 \quad 9 \cdot 8$ 
unnecessary to present a figure to show that Reichel's formula does not fit these data.

When the higher concentrations are considered, it appears that the v.s.t.-concentration curves become asymptotic to the abscissa so that it is not possible to postulate the existence of clearly defined maximum concentrations at which the v.s.t.'s approach zero and be fixed, defined as the temperature giving a v.s.t. of $10 \mathrm{~min}$. Owing to the difficulty of determining with accuracy v.s.t.'s shorter than this, it was urged that little would be lost by this restriction of the data. A similar argument applies in the present case and it may be considered that there is an effective maximum concentration for each temperature, defined as the

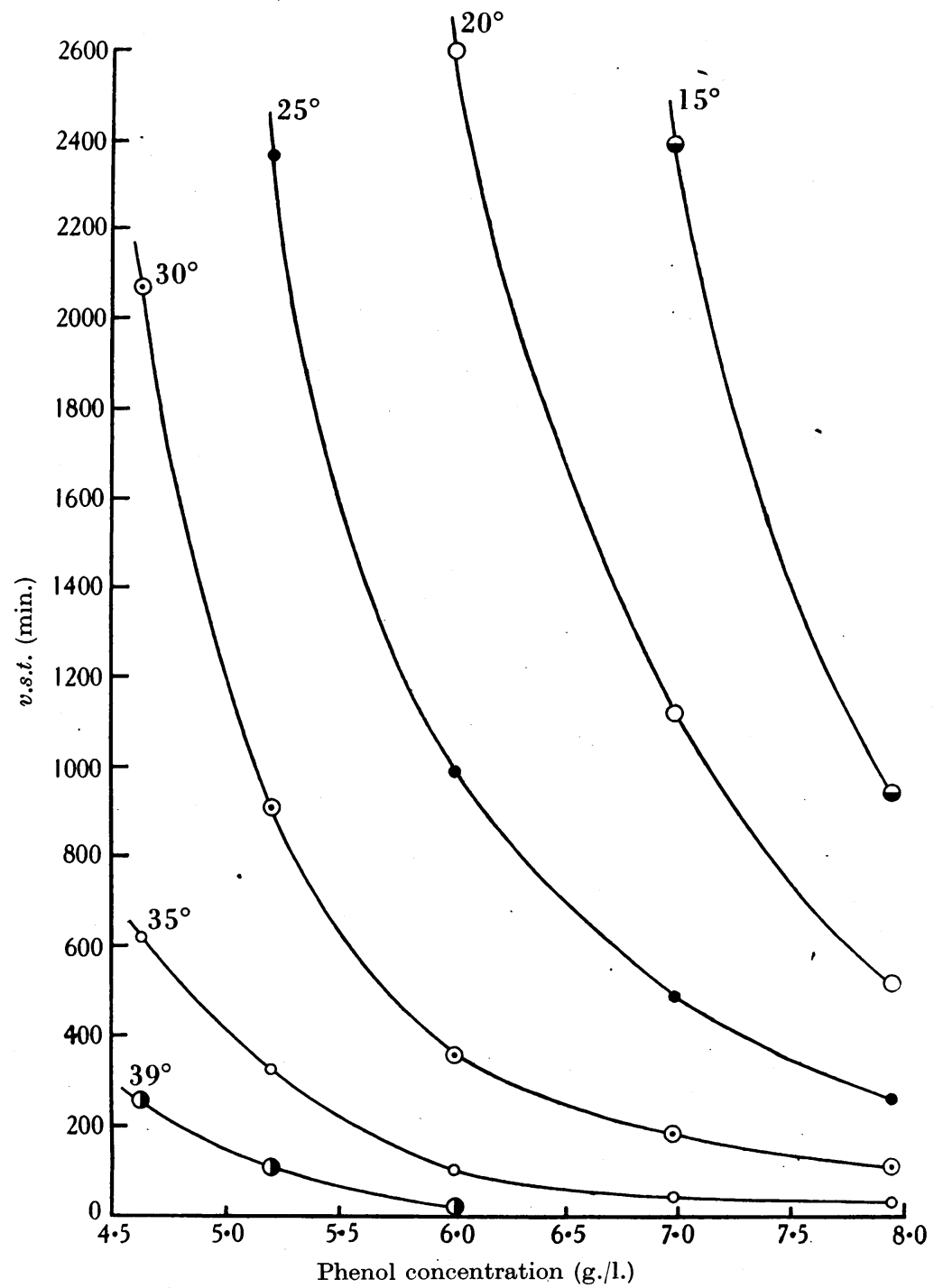

Fig. 1. Showing relation between v.s.t. and phenol concentration at various temperatures.

the disinfections become almost instantaneous. In these circumstances recourse may be had to a device used in the previous paper of this series (Jordan \& Jacobs, 1946b) in an analysis of the relationship between v.s.t. and temperature at constant phenol concentration. There it was suggested that for each concentration a useful maximum temperature might concentration giving a v.s.t. of $10 \mathrm{~min}$. It then becomes possible to regard (v.s.t. -10 ) as a quantity which, at constant temperature, decreases from infinity to zero as the concentration of phenol rises from the minimum $\left(C_{\min }\right)$ to the maximum $\left(C_{\max }\right)$. The logarithm of (v.s.t. -10$)$ should then vary between plus and minus infinity and it is likely that some form of sigmoid curve 
would be realized when $\log ($ v.s.t. -10$)$ is plotted against concentration over the full range. The graphs would probably be asymptotic to ordinates at values of $C_{\mathrm{min}}$ and $C_{\max }$ appropriate to the temperatures chosen. A similar conclusion could equally well be reached from a study of the system of logistic curves representing the relation between $\log ($ v.s.t. -10$)$ and temperature at constant concentration (Jordan \& Jacobs, 1946b). The values for $\log ($ v.s.t. -10$)$ for various concentrations at constant temperature are limit any ordinate would fail to intersect the curves for concentrations above and below certain values. These would then be the maximum and minimum concentrations referred to above, and the $\log ($ v.s.t. -10$)$ against concentration curves must be asymptotic to ordinates placed at these values.

The exact shape of the sigmoid curve required is not known, but in the previous paper of this series the equation of the Pearl-Verhulst logistic curve was successfully applied, as stated above, to curves of

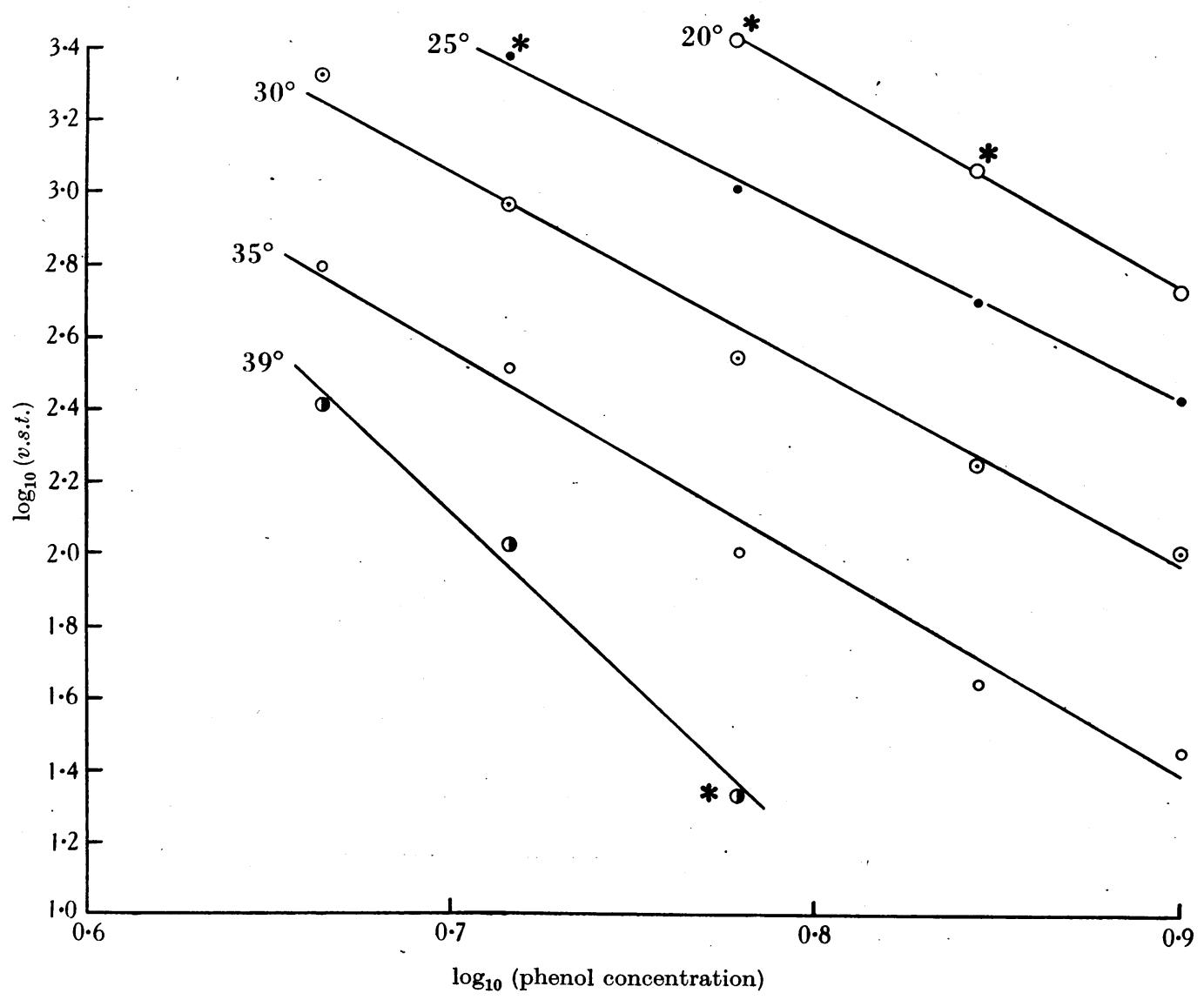

Fig. 2. Showing relation between $\log _{10}$ v.s.t. and $\log _{10}$ (phenol concentration) at constant temperature.

* Point outside experimental range.

given by the points of intersection of these curves with ordinates erected at appropriate positions on the abscissa, i.e., the temperature axis. If sufficient curves were available and these corresponded to successive uniform increments in phenol concentration, it is clear that the intersections by any ordinate would be widely spaced for high values of $\log (v . s . t .-10)$, then more closely spaced and finally widely spaced again as $\log (v . s . t .-10)$ decreased, a condition which must result in a sigmoid curve when $\log ($ v.s.t. -10$)$ is plotted against concentration. Further, it is clear that in the type similar to those under discussion here. The equation to this curve, with symbols appropriate to the present case, is

$$
C-C_{\min .}=\frac{C_{\max }-C_{\min }}{1+e^{k+8 \log _{10}(v . s . t-10)}},
$$

where $C$ is the experimental phenol concentration,

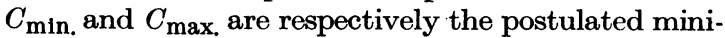
mum and maximum concentrations, and $k$ and $s$ are constants. On re-arranging this formula and taking logarithms this expression becomes converted to 
$\log _{10}\left[\frac{C_{\text {max. }}-C}{C-C_{\text {min. }}}\right]=0 \cdot 4343\left[k+s \log _{10}(\right.$ v.s.t. -10$\left.)\right]$.

This is of the form of the general equation to a straight line and testing the agreement of the data with the logistic formula is simply a matter of finding how closely the lines of regression of $\log _{10}\left[\frac{C_{\max }-C}{C-C_{\min }}\right]$ on $\log _{10}$ (v.s.t. -10$)$ approach to straight lines. But before this can be done values for $C_{\min }$. and $C_{\max }$. must be fixed, and while Fig. 1 gives some indication of certain gives an infinitely large v.s.t. at the chosen temperature, i.e., it is $C_{\mathrm{min}}$, and we can therefore write

$$
T=69 \cdot 83-10 \cdot 75 C_{\mathrm{min}} .
$$

Similarly, from the hyperbolic relationship

$$
\left(T_{\max }-10\right)(C-3)^{0 \cdot 2632}=41 \cdot 53,
$$

which was found to exist between the maximum temperature ( $T_{\max }$ ) and concentration, it is clear that the value of $C$ corresponding to any chosen $T_{\max }$. must be $C_{\text {max }}$, since it is the concentration which would

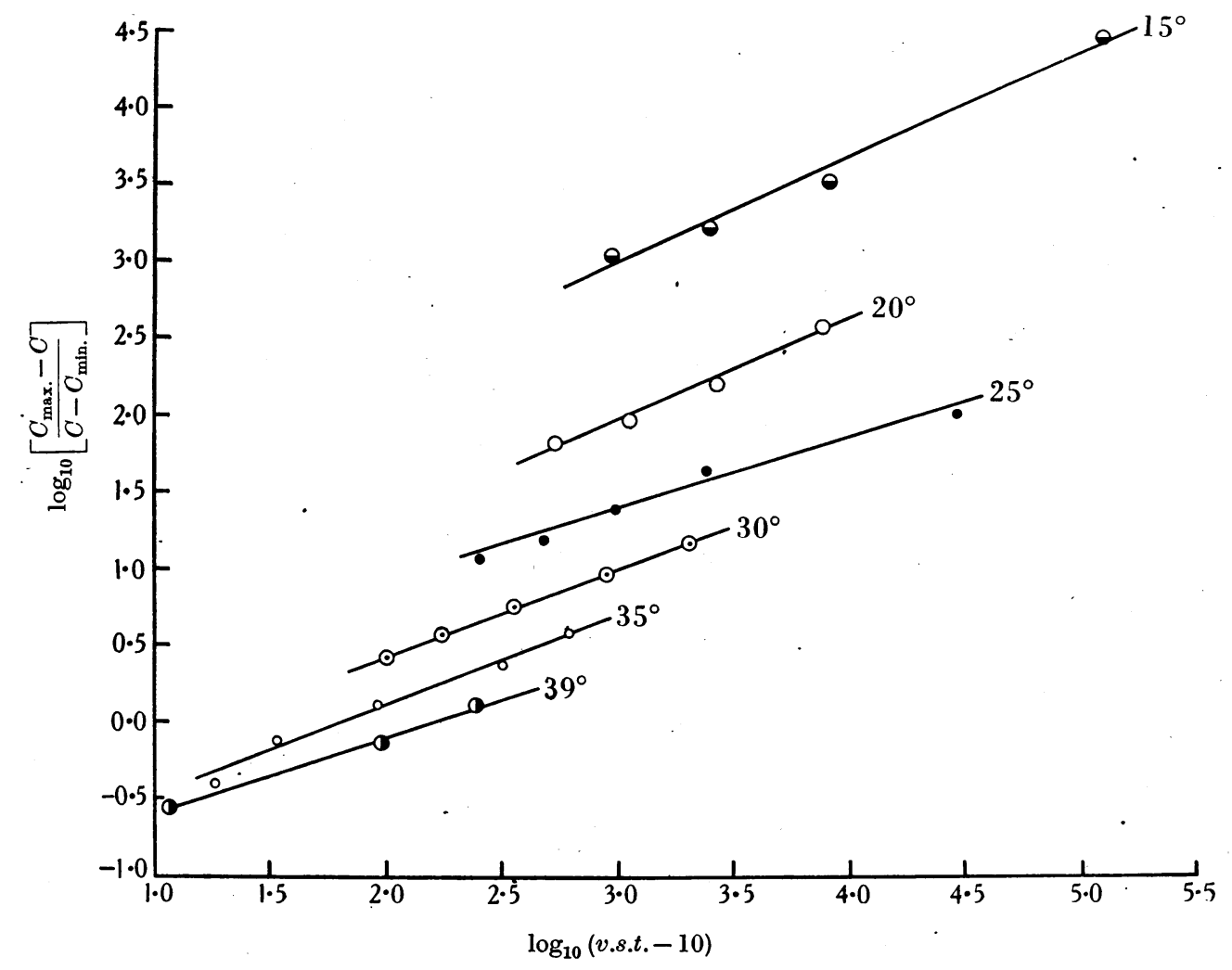

Fig. 3. Showing relation between $\log _{10}\left[\frac{C_{\max }-C}{C-C_{\min .}}\right]$ and $\log _{10}($ v.s.t. -10$)$ at constant temperature.

values of $C_{\min }$ and one value of $C_{\max }$ the remaining values are quite indefinite. Fortunately, it is possible to obtain values for these constants by utilizing the relationships already found to exist between minimum and maximum temperature and phenol concentration (Jordan \& Jacobs, 1946b). The minimum temperature $\left(T_{\mathrm{min}}\right)$ was found to be linearly related to phenol concentration ( $C \mathrm{~g}$./1.) according to the formula

$$
T_{\text {min. }}=69 \cdot 83-10 \cdot 75 \mathrm{C} \text {. }
$$

Looking at this in another way, the value of $C$ corresponding to any value of $T_{\mathrm{min}}$. must be that which give a v.s.t. of $10 \mathrm{~min}$. at that temperature. We have, therefore, that

$$
(T-10)\left(C_{\max }-3\right)^{0.2632}=41.53 .
$$

Table 3 gives the calculated values of $C_{\min }$ and $C_{\max }$ for the temperatures chosen, together with the equations of the corresponding best fitting straight lines based on all the data in Table 1, and the standard errors of the slopes, of these lines. The latter are all small in comparison with the slopes and it may be concluded that the logistic formula provides an adequate expression of the data. Fig. 3, which shows the data of 
Table 1 plotted over the calculated lines corresponding to the equations of Table 3, illustrates the degree of linearity obtained, while in Fig. 4 the actual calculated logistic curves of $\log ($ v.s.t. -10$)$ plotted against phenol concentration are shown.
The values of $C_{\max }$ and $C_{\min }$ given in Table 3 are not necessarily those giving the closest linearity in the relationship between $\log _{10}($ v.s.t. -10$)$ and $\log _{10}\left[\frac{C_{\max }-C}{C-C_{\min }}\right]$, and should rather be regarded as

Table 3. The constants of the logistic equation as fitted to the $\log _{10}(v . s . t .-10)$-concentration data on the basis of a hyperbolic relationship between $C_{\max }$. and temperature

\begin{tabular}{|c|c|c|c|c|c|c|}
\hline $\begin{array}{l}\text { Temp. } \\
{ }^{\circ} \mathrm{C} .\end{array}$ & $\begin{array}{c}C_{\max } \\
\mathrm{g} \cdot / \mathrm{l}\end{array}$ & $\begin{array}{c}C_{\min } . \\
\mathrm{g} . / 1 .\end{array}$ & $\begin{array}{c}\text { Formula of } \\
\text { regression line } \\
* Y=0 \cdot 4343 s x+0 \cdot 4343 k\end{array}$ & $\begin{array}{c}\text { Standard } \\
\text { error of } \\
0 \cdot 4343 s\end{array}$ & $\begin{array}{l}\text { Ratio of } \\
0 \cdot 4343 s \text { to } \\
\text { its standard } \\
\text { error }\end{array}$ & $-k / s$ \\
\hline 15 & 3114 & $5 \cdot 10$ & $Y=0.7051 x+0.8691$ & \pm 0.0549 & $12 \cdot 8$ & $-1 \cdot 233$ \\
\hline 20 & $226 \cdot 5$ & $4 \cdot 63$ & $0.6586 x-0.0033$ & \pm 0.0597 & $11 \cdot 0$ & 0.005 \\
\hline 25 & $50 \cdot 87$ & $4 \cdot 17$ & $0.4721 x-0.0419$ & \pm 0.0429 & $11 \cdot 0$ & $0 \cdot 089$ \\
\hline 30 & $19 \cdot 05$ & $3 \cdot 71$ & $0.5855 x-0.7461$ & \pm 0.0107 & $54 \cdot 8$ & $1 \cdot 274$ \\
\hline 35 & $9 \cdot 88$ & $3 \cdot 24$ & $0.5957 x-1.0746$ & \pm 0.0488 & $12 \cdot 2$ & $1 \cdot 804$ \\
\hline \multirow[t]{3}{*}{39} & $6 \cdot 91$ & $2 \cdot 87$ & $0.4868 x-1.0672$ & \pm 0.0384 & $12 \cdot 7$ & $2 \cdot 192$ \\
\hline & & & Mean $\overline{0 \cdot 5840}$ & & & \\
\hline & & & $0 \cdot 0920$ & & & \\
\hline
\end{tabular}

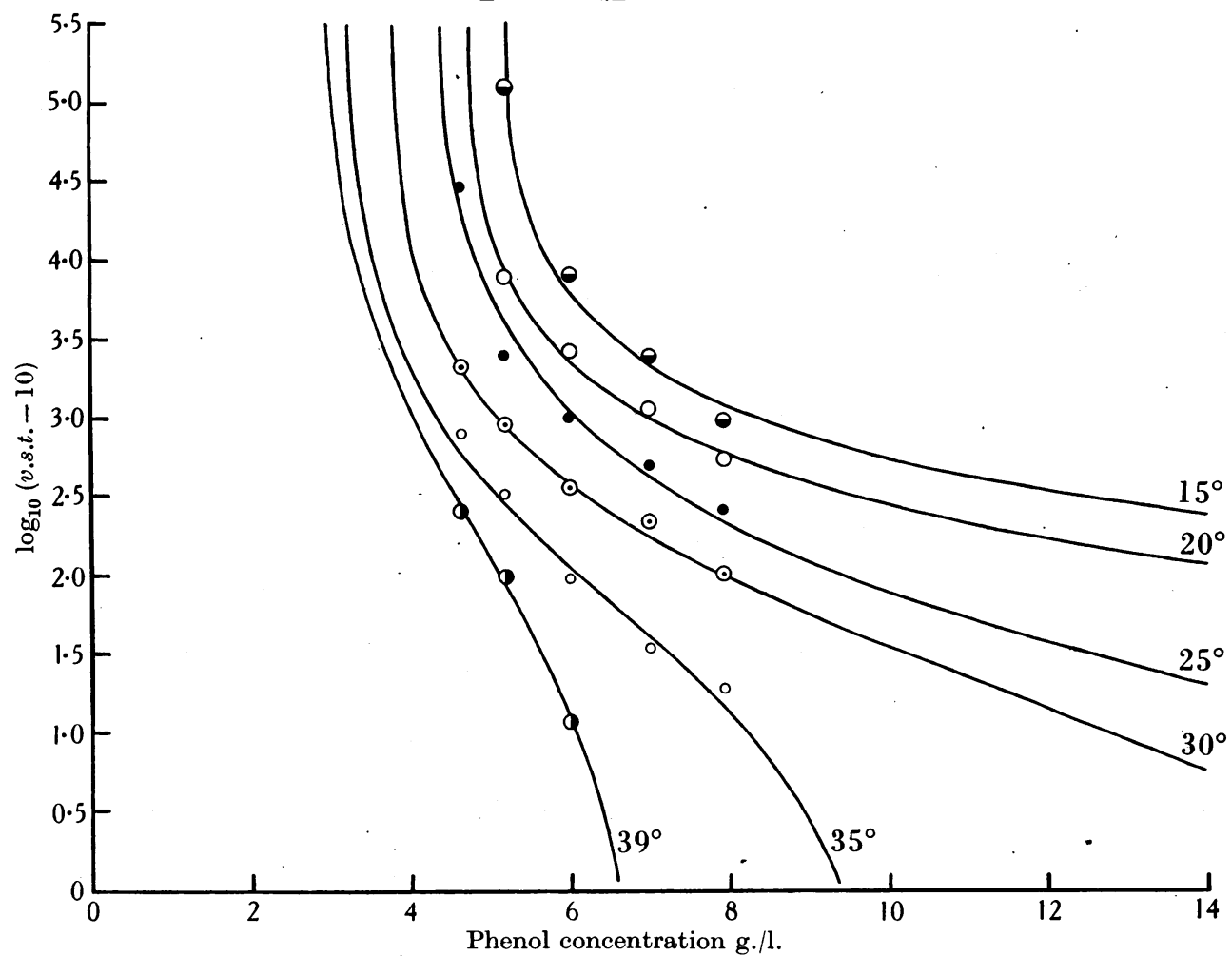

Fig. 4. Showing relation between $\log _{10}($ v.s.t. -10$)$ and phenol concentration.

The equations to these curves may be put in another form which for certain purposes (see below) is more useful. Equation (2) above then becomes

$$
\begin{aligned}
& (v . s . t .-10)=B\left[\frac{C_{\max }-C}{C-C_{\min }}\right]^{\frac{1}{0 \cdot 4343 s}}, \\
& { }_{10} B=-k / s .
\end{aligned}
$$
where $\log _{10} B=-k / s$. tentative values which have, nevertheless, proved very successful. Indeed, inspection of the graphs in Figs. 3 and 4 leads one to expect that an improvement could be effected particularly at $15^{\circ}$ and $20^{\circ} \mathrm{C}$. and actual trials have shown that improvement in fit can be obtained by choosing slightly different values for these 
constants. The data, for all except the two highest temperatures, are concentrated near the upper bends of the logistic curves of $\log (v . s . t .-10)$ plotted against phenol concentration (Fig. 4) so that changes in $C_{\mathrm{min}}$. will in general have more effect on the closeness of fit than equal changes in $C_{\max }$. The arrangement of the points is, however, such that the calculated values of $C_{\mathrm{min}}$ in Table 3 are very close to the improved values which can be obtained by trial, keeping to the same values for $C_{\max }$. Therefore, it has not been considered necessary to attempt to find by trial the 'best' values of $C_{\mathrm{min}}$, especially as the values of $C_{\max }$ may themselves be in error. The relationship between $T_{\max }$ and concentration from which these values of $C_{\max }$. were derived (see Table 3 ) was a purely empirical one based on values of $T_{\max }$. chosen by inspection from freehand graphs of $\log (v . s . t$.$) plotted against temperature$ (Jordan \& Jacobs, 1946b). While these values for $T_{\max }$. were adequate for their purpose and could not have been greatly in error, they may not have led to the most useful relationship between $T_{\max }$ and concentration. Actually, although the assumed relationship was hyperbolic, a linear relation would have been more useful, as the following considerations show.

It may be recalled that the relationship between $\log (v . s . t$.$) and temperature was found to be well$ expressed by the logistic formula which could be put in the form

$$
\text { (v.s.t. }-10)=A\left[\frac{T_{\max }-T}{T-T_{\min }}\right]^{\frac{1}{0.4343 r}},
$$

and this has an obvious similarity to equation (7) above. Now, by combining equations (3) and (4) an expression for $T-T_{\mathrm{min}}$. can be derived, and this is found to be

$$
T-T_{\min .}=10 \cdot 75\left(C-C_{\min .}\right) .
$$

This can be substituted in equation (8). If a linear relationship also existed between $T_{\max }$ and concentration, we should obtain a similar expression for direct proportionality between $T_{\max }-T$ and $C_{\max }-C$ and on substituting this in equation (8) the latter would be converted into a form identical with that of equation (7) which has already been found to express the connexion between v.s.t. and concentration. However, the assumed relationship (equation (5)) was hyperbolic and the equation connecting $T_{\max }-T$ with $C_{\max }-C$ derived from expressions (5) and (6) is

$$
\begin{aligned}
& T_{\max }-T \\
& \quad=41 \cdot 53\left[(C-3)^{-0.2632}-\left(C_{\max .}-3\right)^{-0.2632}\right],
\end{aligned}
$$

which does not enable equation (8) to be converted into equation (7) but gives a very unwieldy formula. Since obviously it is desirable that both lines of approach to the tripartite relationship between v.s.t., concentration and temperature should lead to related formulae, preferably of a comparatively simple type such as that of equation (7), it becomes necessary to re-examine the connexion between $T_{\max }$ and concentration. If it be assumed that there is a linear relationship between these two variables, the equation of the best straight line to fit the data is

$$
T_{\max }=55 \cdot 58-2 \cdot 253 C .
$$

The slope of the line has a standard error of $\pm 0 \cdot 3156$ or rather less than one-seventh of the slope. The closeness of fit to the straight line is naturally not so good as to the hyperbola (Jordan \& Jacobs, 1946b), but it must be remembered that the values of $T_{\max }$. were originally chosen from freehand curves. The relation between temperature and $C_{\max }$. then becomes

$$
T=55 \cdot 58-2 \cdot 253 C_{\max }
$$

Table 4 gives the new values of $C_{\max }$ and it will be seen that those corresponding to low temperatures are reduced much below those of Table 3 , but those for the higher temperatures of $35^{\circ}$ and $39^{\circ} \mathrm{C}$. are not greatly affected. Table 4 also gives the equations of the best fitting lines of regression of $\log \left[\frac{C_{\max .}-C}{C-C_{\min }}\right]$ on $\log (v . s . t .-10)$ based on these new values of $C_{\max }$, together with the standard errors of the slopes of these lines. There has been some improvement in the fit for the two lowest temperatures while at other temperatures the fit is rather less good. On the whole the new values of $C_{\max }$. have not brought about any great change in the picture and the linear relationship (11) between $T_{\max }$ and concentration would seem to be equally as good as the hyperbolic for deriving values of $C_{\max }$.

From formulae (11) and (12) we find that

$$
T_{\max .}-T=2 \cdot 253\left(C_{\max }-C\right)
$$

and making the appropriate substitutions in equation (8) we have

$$
\text { (v.s.t. }-10)=A\left[\frac{2 \cdot 253\left(C_{\max .}-C\right)}{10 \cdot 75\left(C-C_{\min }\right)}\right]^{\frac{1}{0 \cdot 4343 r}},
$$

and this is evidently of the same form as equation (7) above.

The question then arises as to the identity of the two formulae, i.e., whether the values of the constants are the same, but here a difficulty arises because while equation (8) was shown to apply well to the data for a single phenol concentration there was some doubt as to whether the constants of the equation were identical at all concentrations. Whilst $r$ might have been fixed, $A$ may have varied. The values of the constants for formula (7) must also, therefore, be examined to see whether they vary for different temperatures or whether one formula can be found to apply to all temperatures. From Table 3 it appears that apart from a possible slight tendency to fall with rising temperature the values of $0.4343 \mathrm{~s}$ may be regarded as fluctuating about a mean value of 0.5840 with a standard deviation of 0.0920 and a coefficient 
of variation of $15 \cdot 75 \%$. The values in Table 4 fluctuate similarly, but rather more widely and with a somewhat greater tendency to fall with rising temperature, about a mean of $0 \cdot 6455$, the standard deviation being $0 \cdot 1384$ and the coefficient of variation $21.44 \%$. The two means do not differ significantly and it may be noted that both values, especially the latter, are exceedingly close to that of 0.6377 (S.D. $=0.0865$ ) obtained for $0.4343 r$ in the formula connecting v.s.t. with temperature at constant phenol concentration (Jordan \& Jacobs, 1946b). The values of $-k / s$ in Table 3 rise consistently and markedly with increasing temperature, but those in Table 4 are well-nigh constant, the mean being $2 \cdot 100$ with a standard deviation of $0 \cdot 1573$ and a coefficient of variation of $7.49 \%$. The very slight tendency that these latter valuesshow to decrease with rising temperature can hardly be real. The relative constancy of the values of both $0 \cdot 4343 s$ and $-k / s$ in may have been the case. Although these values could not in general have been greatly in error, extrapolation in order to find $T_{\max }$ for low phenol concentrations may have given values which were much too large. At all events, if the mean values of 1566 for $A$ and 1.5681 for $1 / 0 \cdot 4343 r$ (given in the paper quoted above) are applied to formula (14) the latter becomes

$$
(\text { v.s.t. }-10)=138 \cdot 2\left[\frac{C_{\max }-C}{C-C_{\min }}\right]^{1.5681} \text {, }
$$

which is practically identical with equation (15) derived directly from the data relating v.s.t. to concentration at constant temperature. Considerable unification of the relationships between v.s.t., temperature and concentration has thus been achieved.

The constant $1 / 0 \cdot 4343 s$ in formula (7) partakes of the nature of a concentration exponent and it is a particularly satisfactory one as not only may its value

Table 4. The constants of the logistic equation as fitted to the $\log _{10}(v . s . t .-10)$-concentration data on the basis of a linear relationship between $C_{\max }$ and temperature

\begin{tabular}{|c|c|c|c|c|c|c|c|}
\hline $\begin{array}{l}\text { Temp. } \\
{ }^{\circ} \mathrm{C} .\end{array}$ & $\begin{array}{l}C_{\max } \\
\text { g./l. }\end{array}$ & $\begin{array}{l}C_{\min .} \\
\text { g./l. }\end{array}$ & $* Y=$ & $\begin{array}{l}\text { Formula of } \\
\text { regression line } \\
=0 \cdot 4343 s x+0 \cdot 4343 k\end{array}$ & $\begin{array}{l}\text { Standard } \\
\text { error of } \\
0 \cdot 4343 s\end{array}$ & $\begin{array}{c}\text { Ratio of } \\
0 \cdot 4343 s \text { to } \\
\text { its standard } \\
\text { error }\end{array}$ & $-k / s$ \\
\hline 15 & $18 \cdot 01$ & $5 \cdot 10$ & & $=0.7520 x-1 \cdot 7448$ & \pm 0.0434 & $17 \cdot 3$ & $2 \cdot 320$ \\
\hline 20 & $15 \cdot 79$ & $4 \cdot 63$ & & $0 \cdot 7646 x-1 \cdot 7280$ & \pm 0.0476 & $16 \cdot 1$ & $2 \cdot 266$ \\
\hline 25 & $13 \cdot 57$ & $4 \cdot 17$ & & $0.5485 x-1.0708$ & \pm 0.0631 & $8 \cdot 7$ & 1.952 \\
\hline 30 & $11 \cdot 35$ & $3 \cdot 71$ & & $0 \cdot 7169 x-1 \cdot 4941$ & \pm 0.0292 & $24 \cdot 6$ & $2 \cdot 084$ \\
\hline 35 & $9 \cdot 13$ & $3 \cdot 24$ & 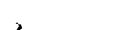 & $0 \cdot 6791 x-1 \cdot 3581$ & \pm 0.0719 & $9 \cdot 5$ & $2 \cdot 000$ \\
\hline \multirow[t]{3}{*}{39} & $7 \cdot 36$ & $2 \cdot 87$ & & $0.4118 x-0.8132$ & \pm 0.0442 & $9 \cdot 3$ & $1 \cdot 975$ \\
\hline & & & Mean & $0 \cdot 6455$ & & Mean & $2 \cdot 100$ \\
\hline & & & S.D. & $0 \cdot 1384$ & & S.D. & $0 \cdot 1573$ \\
\hline
\end{tabular}

Table 4, which are based on a linear relation between $T_{\max }$ and phenol concentration, suggests that this is to be preferred to the hyperbolic formula as a means of settling the values of $C_{\max }$, as it leads to the establishment of a single formula valid for all temperatures for the relation between v.s.t. and concentration at constant temperature. Using the mean values of $0.4343 s$ and $-k / s$ shown in Table 4 , we find that $1 / 0 \cdot 4343 s=1.5492$ and $B$, which is the antilogarithm of $-k / s$, is $125 \cdot 9$. Equation ( 7 ) accordingly becomes .

$$
(v . s . t .-10)=125 \cdot 9\left[\frac{C_{\max }-C}{C-C_{\min } .}\right]^{1 \cdot 5492} \text {. }
$$

As it has proved possible to find a value for $B$ applicable to all temperatures, it is possible that $A$ in formulae (8) and (14) might also have a fixed value for all phenol concentrations. Previously it had been suggested (Jordan \& Jacobs, 1946b) that its value must fall with decreasing concentration, but the evidence then available was based on certain chosen values of $T_{\max }$ which may not have been the best. As shown above, there are good grounds for supposing that this be constant for all temperatures, but it has been derived by a method of treating disinfection times which takes account of the existence of a minimum effective concentration and may, therefore, be expected to apply to all concentrations while the exponent employed hitherto must increase when the minimum concentration is approached. Application of the formula $C^{n} \times t=K$ must lead to values for the v.s.t. which, at concentrations near the minimum, are far too small and this state of affairs was actually found to exist in an extensive series of experiments conducted at $35^{\circ}$ C. (Jordan \& Jacobs, 1944, 1945). Using the logistic formula and the values of $C_{\max }$ and $C_{\min }$. given in Tables 3 and 4, the v.s.t. calculated for $3 \cdot 48 \mathrm{~g}$. $/ 1$. is considerably too high, i.e., these limits lead to an over-correction. This indicates that $C_{\mathrm{m}}$. is too high and if this value be decreased to $3.00 \mathrm{~g}$./l. while $C_{\max }$. remains at $9 \cdot 13 \mathrm{~g}$./l. the discrepancy almost disappears; the calculated value for the v.s.t. is $3760 \mathrm{~min}$. as compared with the experimental value of $3715 \mathrm{~min}$. The new concentration exponent is, therefore, to be preferred to the old, especially as the formula involved 
in its use gives an indication of the effective concentration range of the germicide. However, it must be stressed that the logistic formula has been employed only as a convenient one of the right type yielding relatively simple relationships between the variables examined. It does not necessarily give the closest possible fit to the results, but in view of its comparative simplicity and the success which has been achieved in fitting the present data its use may be considered to be justified.

\section{SUMMARY}

1. Calculated values of the virtual sterilization time (v.s.t.) have been used in an analysis of the effect of temperature on the concentration exponent of the reaction between phenol and Bact. coli cultures under rigidly standardized conditions.

2. Within the experimental range of temperature and concentration employed, the exponent calculated from the formula $C^{n} \times t=K$ was satisfactorily constant from $20^{\circ}$ to $35^{\circ} \mathrm{C}$, , but it apparently increased at $39^{\circ} \mathrm{C}$.

3. This formula is unsatisfactory in that it cannot apply to concentrations near the threshold value without a change in the value of $n$. Accordingly, a new method of treating these data has been suggested which makes allowance for this threshold or minimum concentration. A corresponding 'maximum' concentration has been arbitrarily fixed at which the v.s.t. is $10 \mathrm{~min}$. and the value of (v.s.t. - 10) thus varies from infinity to zero between these concentration limits.

4. Sigmoid curves asymptotic to ordinates at the minimum and maximum concentrations must be obtained when $\log (v . s . t .-10)$ is plotted against concentration and the equation of the Pearl-Verhulst logistic curve, which is of the required type, has been used successfully to express the relationship between v.s.t. and concentration at constant temperature.

5. One of the constants of this formula partakes of the nature of a concentration exponent and this may be constant over the full effective concentration range and for all temperatures.

6. The logistic formula relating v.s.t. to concentration at constant temperature is closely related to that connecting $v$.s.t. with temperature at constant concentration. The two formulae become interconvertible if certain assumptions are made concerning the relationships between the minimum and maximum concentrations and temperature.

\section{REFERENCES}

Jordan, R. C. \& JAcobs, S. E. (1944). J. Hyg., Camb., 43, 363.

Jordan, R. C. \& JACOBS, S. E. (1945). J. Hyg., Camb., 44, 210.

JoRdAN, R. C. \& JAcobs, S. E. (1946a). J. Hyg., Camb., 44, 243.

Jordan, R. C. \& JAcobs, S. E. (1946b). J. Hyg., Camb., 44249.
Pearl, R. (1930). Medical Biometry and Statistics. London: Saunders.

RaHn, O. (1932). Physiology of Bacteria. Philadelphia: Blakiston.

Reichel, H. (1909). Biochem. Z. 22, 149.

Tilley, F. W. (1939). J. Bact. 38, 499.

(MS. received for publication 13. vIII. 45.-Ed.) 